\title{
Modeling two-dimensional magnetic domain patterns
}

\author{
J. R. Iglesias, ${ }^{1}$ S. Gonçalves, ${ }^{1}$ O. A. Nagel,${ }^{2}$ and Miguel Kiwi ${ }^{3}$ \\ ${ }^{1}$ Instituto de Física, Universidade Federal do Rio Grande do Sul, Caixa Postal 15051, 91501-970 Porto Alegre RS, Brazil \\ ${ }^{2}$ Departamento de Física, Universidad Nacional del Sur, Av. Alem 1253, 8000 Bahía Blanca, Argentina \\ ${ }^{3}$ Facultad de Física, Pontificia Universidad Católica de Chile, Casilla 306, Santiago, Chile 6904411
}

(Received 29 August 2001; published 24 January 2002)

\begin{abstract}
Two-dimensional magnetic garnets exhibit complex and fascinating magnetic domain structures, like stripes, labyrinths, cells, and mixed states of stripes and cells. These patterns do change in a reversible way when the intensity of an externally applied magnetic field is varied. The main objective of this contribution is to present the results of a model that yields a rich pattern structure that closely resembles what is observed experimentally. Our model is a generalized two-dimensional Ising-like spin-1 Hamiltonian with long-range interactions, which also incorporates anisotropy and Zeeman terms. The model is studied numerically by means of Monte Carlo simulations. Changing the model parameters, stripes, labyrinth, and/or cellular domain structures are generated. For a variety of cases we display the patterns and determine the average size of the domains, the ordering transition temperature, specific heat, magnetic susceptibility, and hysteresis cycle. Finally, we examine the reversibility of the pattern evolution under variations of the applied magnetic field. The results we obtain are in good qualitative agreement with experiment.
\end{abstract}

DOI: 10.1103/PhysRevB.65.064447

PACS number(s): 75.70.Rf, 89.75.Kd, 61.43.Bn

\section{INTRODUCTION}

Magnetic domains in magnetic garnet films are interesting in themselves because of the rich and complex pattern formation they display; stripes, cells, labyrinths, ordered hexagonal lattices, disordered froth, pentagonal structures along the front between the ordered lattice and the disordered froth, and mixed states of stripes and cells are some of the features observed experimentally. ${ }^{1-4}$ In addition, these patterns are quite universal, since they are similar to those observed in soap froth and in the annealing process of polycrystalline materials. ${ }^{5,6}$ Moreover, the transitions are driven by stress induced by an applied magnetic bias field $\mathbf{H}$, a handy control parameter, which plays a role analogous to temperature and/or pressure in the melting transition of solids.

Originally the structure of magnetic domains in ultrathin magnetic garnet films attracted attention due to their potential application as magnetic bubble memories. ${ }^{1}$ In fact, the size and thermal stability of the domains are crucial to optimize recording performance. Due to the interesting physics related to these technological problems, significant basic research efforts have been carried out lately. ${ }^{7-9}$ The most extensively investigated systems are single-crystal ferromagnetic thin films of the FeYBiGdGaO garnet type. They are 5-7 $\mu \mathrm{m}$ thick and exhibit a considerable anisotropy along the direction perpendicular to the film, so that the magnetization remains along this axis except within the Bloch walls that separate opposite direction domains. These Bloch walls are of the order of $0.1 \mu \mathrm{m}$ thick. Different types of stable and metastable domain structures are generated in such materials, ${ }^{4,9-11}$ depending on the transient magnetic fields applied during the thermomagnetic cycling of the sample. Eye-appealing examples of the most common structures observed experimentally were obtained by Albuquerque and are found in Ref. 10. The most prototypical examples, displayed in Fig. 1, can be grouped into several categories: parallel stripes [Fig. 1(a)], mixed states of labyrinths and bubbles [Fig. 1(b)], pure labyrinths [Fig. 1(c)], and cell structures with a strong labyrinth component, as illustrated in Fig. 1(d)

Often the bubble structure looks like soap froth, which originated the denomination "magnetic foam." These magnetic foams are generated by cooling the sample, initially at a temperature $T$ larger than the critical one $T_{c}$, to $T<T_{c}$ in the presence of a weak magnetic field perpendicular to the plane of the film. The magnetic bubble patterns result from a balance between the demagnetizing energy (like long-range dipolar interactions) and the energy required to create Bloch walls. This balance originates an equilibrium domain size, which evolves as a function of the strength of the applied magnetic field. When this external field is increased, the bubble structure coarsens and large bubbles, surrounded by smaller ones, are observed, while the total number of bubbles decreases.

Another intriguing feature of these foams is the memory effect. It consists in a reversible change of the foam topology under a cyclic variation of the applied magnetic field. For example, the bubble configuration observed under an applied magnetic field evolves into a labyrinth pattern at zero field,

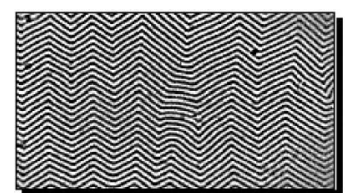

(a)

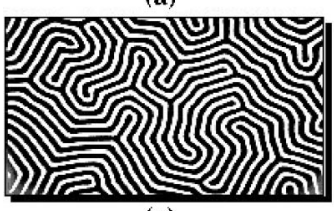

(c)

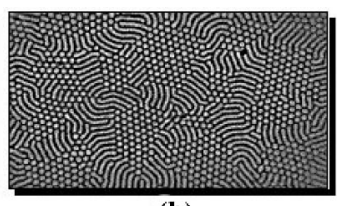

(b)

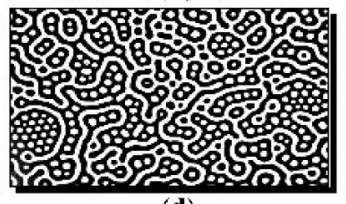

(d)
FIG. 1. Stable domain structures observed experimentally by Albuquerque (Ref. 10) in a magnetic garnet. Obtained by varying the combination of perpendicular to the film and in plane applied magnetic fields. 
but the system returns to the previous macroscopic configuration when the magnetic field is restored. It is the stability of the threefold vertices that seems to be responsible for this interesting memory effect., ${ }^{2,4}$

Over the years significant effort has been devoted to the understanding of this rich and complex behavior. ${ }^{1,2}$ For the particular case of magnetic froth Weaire et al. ${ }^{11}$ proposed a description based on an analogy with two-dimensional soap froth, including surface and compressibility energy terms. Sampaio et al. ${ }^{8}$ proposed a model Hamiltonian to describe the formation of magnetic domains in strongly anisotropic magnetic thin films, on the basis of a ferromagnetic Ising Hamiltonian, including long-range (antiferromagnetic) dipole-dipole interactions and an external magnetic field.

In this contribution we put forward an alternative model, addressing the problem of the magnetic domain pattern formation by means of a generalized spin Hamiltonian. More precisely, we consider a three-state spin-1 Ising Hamiltonian, with competing long-range interactions, and we also include the anisotropy energy and an external field.

This paper is organized as follows: after this Introduction, in Sec. II we present the model and the simulation technique. In Sec. III the results reached by implementing our model are presented. The paper is closed in Sec. IV, where we provide a summary and draw conclusions.

\section{HAMILTONIAN AND SIMULATION}

\section{A. Hamiltonian}

We model the system by a generalized spin-1 Ising-like Hamiltonian on a square lattice. The interaction between nearest-neighboring spins is ferromagnetic and long-range competing dipolar interactions, between the $n$ nearestneighbor sites $(n>1)$, are incorporated. We also include the magnetic field and the anisotropy. Analytically our model Hamiltonian is

$$
\begin{aligned}
H= & -\frac{1}{2} \sum_{i, j}{ }^{\prime} J_{i j} S_{i}^{(z)} S_{j}^{(z)}-\sum_{i}\left[A\left(S_{i}^{(z)}\right)^{2}+\mathbf{H}_{l} S_{i}^{(z)}\right. \\
& \left.+\mathbf{H}_{t}\left\{1-\left(S_{i}^{(z)}\right)^{2}\right\}\right],
\end{aligned}
$$

where the parameters $J_{i j}$ are the exchange coupling constants between the spin on the $i$ site and its first $n$ nearestneighboring spins, located on sites $j$. Thus, $J_{i j}>0\left(J_{i j}<0\right)$ implies ferromagnetic (antiferromagnetic) coupling. The anisotropy constant is defined such that $A>0$ favors a spin orientation perpendicular to the plane of the sample. $\mathbf{H}_{l}$ and $\mathbf{H}_{t}$ are the intensity of the longitudinal and transverse external magnetic fields, respectively, and the $i$ and $j$ summations are performed over $N(N-1)$ lattice sites, since the primed summation implies that the self-interaction terms $i=j$ are excluded. The transverse magnetic field and the anisotropy play a similar role, but while $A$ may be considered as a microscopic characteristic of the system, $\mathbf{H}_{l}$ and $\mathbf{H}_{t}$ are externally controlled parameters.

If one chooses for the coupling constants $J_{i j}$, the dipolar interaction a square-angled labyrinth is generated, as in Ref. 8. Instead, we adopt a long-range oscillatory potential of the
RKKY type. This way, and although the system is not metallic, use of an RKKY-like expression provides a set of oscillating coupling constants that depends on a single parameter $k$. The analytical expression we propose for $J_{i j}$ is given by

$$
J_{i j}=J_{0} \frac{\cos \left(k r_{i j}\right)}{\left(k r_{i j}\right)^{3}},
$$

where $r_{i j}=\left|\vec{r}_{i}-\vec{r}_{j}\right|$ is the distance between sites $i$ and $j$. In the numerical calculations we consider interactions up to the seventh nearest neighbor and for convenience we adopt as our unit of energy, here and throughout, the first-neighbor exchange constant $\Gamma$, defined by

$$
\Gamma=J_{0} \frac{\cos (k a)}{(k a)^{3}}=1,
$$

where $a$ is the lattice parameter. This type of coupling guarantees that the interactions between nearest neighbors are always ferromagnetic and of the same strength, while the magnitude and sign of the coupling with the rest of the neighbors depend on $k$.

Thus, our Hamiltonian differs from previous proposals, like the one studied by Sampaio et al., ${ }^{8}$ in at least three important aspects. We adopt (i) spin 1, instead of spin $\frac{1}{2}$, so that the domain walls may adopt a finite width, since spin $\frac{1}{2}$ implies abrupt transitions from one domain to its neighboring one; (ii) a long-range interaction that is not purely antiferromagnetic and decays as $r_{i j}^{-3}$; and (iii) magnetic anisotropy is incorporated. As will become clear hereafter, the inclusion of these three effects leads to significant qualitative and quantitative differences in the results, and allows for a better description of experimental observations.

\section{B. Simulation procedure}

All of our calculations were performed on an $N \times N$ lattice, with $N=200$, using a conventional Monte Carlo (MC) procedure to update the lattice spins at various temperature, $T$, and applied field, H, values. One Monte Carlo step (MCS) consists in the successive updating of $N^{2}$ spins chosen at random, following the Metropolis procedure. Temperatures and energies are measured in units of $\Gamma=1$, and $k$ in units of the inverse lattice parameter $a^{-1}$.

Two different processes were investigated: quenching and relaxation. (a) Quenching: In order to quench the system the lattice temperature $T$ is raised to infinity (all spin states generated at random) and at the first Monte Carlo step $T$ is reduced to a very low value $(T=0.005)$, after which the system is allowed to evolve for 1000 MCS. This way the system is prevented from exploring most of the available energy landscape and quite probably it is quenched into a metastable minimum energy state, different from the lowest minimum. (b) Relaxation: Thermal relaxation is achieved by slowly varying the temperature from a large initial value $(T=5)$ to a final temperature $T=0$, in intervals of $\Delta T$ $=0.05$. To be certain that thermal relaxation is indeed achieved we run 1000 MCS after every temperature step; 
therefore, for a typical thermal relaxation run a total of $\approx 10^{5}$ MCS are required, which is equivalent to $4 \times 10^{9}$ individual spin flips.

During these processes, and as will be described in detail in Sec. III, several thermodynamic properties and parameters of the system are monitored: the energy $E$, the magnetization $M$, and the average domain size $\Lambda$ are evaluated during the quenching process; along with the relaxation process the specific heat $C$ and the magnetic susceptibility $\chi$ were are also monitored, as a function of $T$. In the latter case we perform an average over 20 different initial states of the system in order to decrease the thermal fluctuations. The domain size $\Lambda$ was evaluated by means of a Hoshen-Kopelman ${ }^{13,14}$ type algorithm. This modified algorithm computes the cluster size, identifying all clusters, calculating their surface and degeneracy, and then evaluating the average cluster size. The specific heat $C$ is calculated by means of the energy fluctuations ${ }^{12}$ and is given by

$$
C=\frac{\left\langle E^{2}\right\rangle-\langle E\rangle^{2}}{\left(k_{B} T\right)^{2}}
$$

Finally, the spin susceptibility $\chi$ at zero field is obtained from the magnetization fluctuations and given by

$$
\chi=\frac{\left\langle M^{2}\right\rangle-\langle M\rangle^{2}}{k_{B} T} \text {. }
$$

\section{RESULTS}

\section{A. Model parameters}

The first part of our work was devoted to search for an adequate set of parameters that generates magnetic domain patterns like the ones observed in magnetic foam experiments, ${ }^{9,10}$ and displayed in Fig. 1. The procedure we employ is the following: we adopt a value of $k$ that in turn determines the values of the exchange parameters $J_{i j}$ up to the seventh nearest neighbor without including anisotropy $(A=0)$ and for zero applied magnetic field $(\mathbf{H}=0)$. Next, the system is quenched from $T \rightarrow \infty$ to a very low $T$ value $(T=0.005)$. At the end of the run we keep or discard the choice of parameters by visual inspection. After selecting some interesting values of $k$ we varied the anisotropy $A$. The $k$ values we finally chose, following the above outlined scheme, yield the long-range exchange functions illustrated in Fig. 2.

All the configurations are obtained after 1000 MCS, as explained in Sec. II B. This number of steps is far larger than what is required to reach a steady state. To stress this fact we display in Fig. 3 the domain size $\Lambda$ versus the first 100 MCS. It is clearly noticed that after the first 30 MCS the system reaches steady-state values. It is also apparent that stripes and labyrinths are significantly smaller than the average cell dimension. Along with $\Lambda$, the total energy $E$ and the magnetization $M$ were also computed, but are not displayed graphically here as their time variation is similar to that of $\Lambda$. Thus, all in all it is evident from the behavior of these quantities,

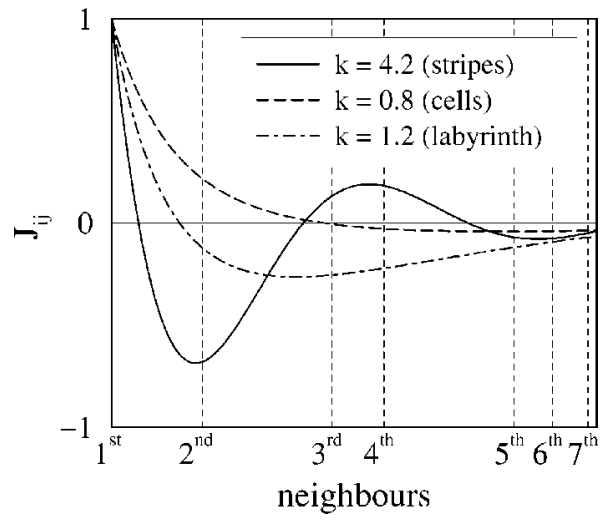

FIG. 2. Long-range RKKY-like dipolar potentials used in the simulations.

and from visual inspection of Fig. 3, that the system reaches steady-state (metastable) equilibrium after relatively few MCS.

The domain structures generated, after quenching, give rise to three different patterns: stripes, labyrinths, and cells. In all cases negative values of the anisotropy $A<0$ were adopted, to favor the presence of domain walls ( $S=0$ sites). These sites emerge preferentially between opposite magnetization domains, since they lower the energy cost of growing a domain wall. Figure 4 exhibits snapshots of the three types of domain structures obtained after quenching. They correspond to the following sets of parameters: (i) $k=4.2$ and $A$ $=-0.5$ (stripes), (ii) $k=0.8$ and $A=-0.1$ (cells), and finally (iii) $k=1.2$ and $A=-0.8$ (labyrinths).

As stated in Sec. II these patterns emerge from the competition between the ferromagnetic first-neighbor exchange interaction $\Gamma>0$ and the long-range oscillating interactions given by Eq. (2.2). For the stripe structure $[k=4.2$, Fig. $4(\mathrm{a})]$ the second-neighbor exchange constant is antiferromagnetic and comparable in magnitude with the nearest-neighbor one, while the third and fourth are ferromagnetic, leading to the formation of stripes of alternating spin orientation. This way, two frustrated couplings between nearest neighbors are compensated by four pairs of satisfied second-neighbor interactions. The labyrinth case $(k=1.2)$, illustrated in Fig. 4(c), is also a consequence of competing interactions between the ferromagnetic first-neighbor coupling and the rest, all of

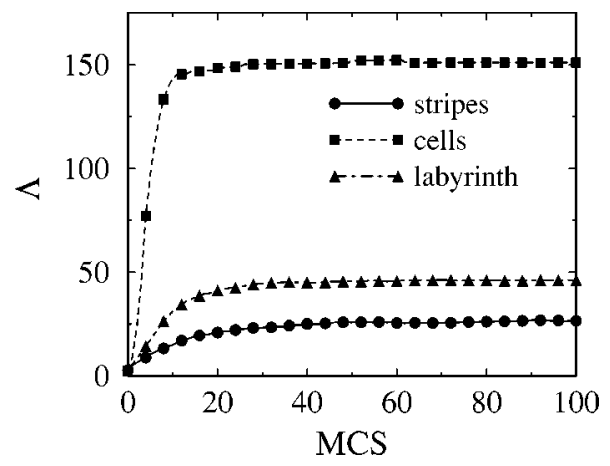

FIG. 3. Average domain size $\Lambda$ vs number of Monte Carlo steps (MCS). 
(a)

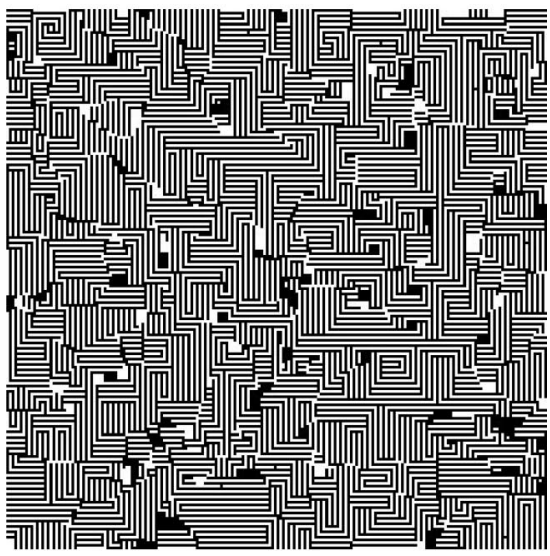

(b)

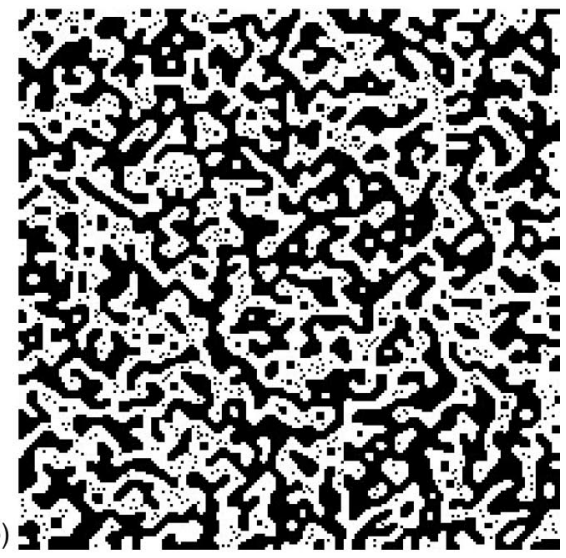

(c)

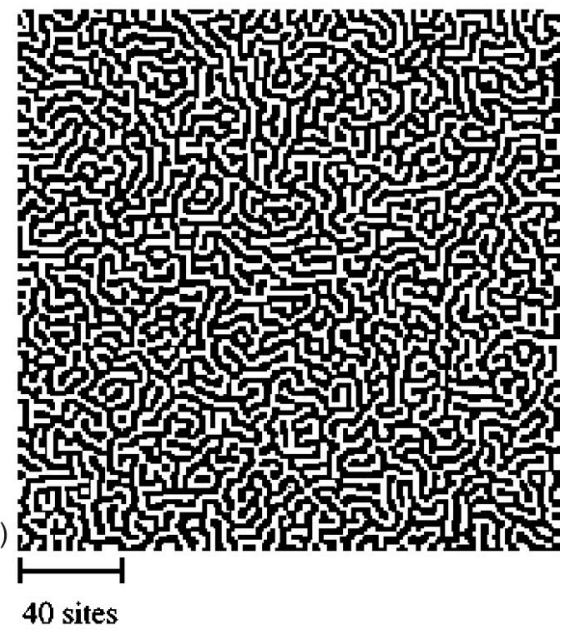

FIG. 4. Snapshots of the three types of domain structure obtained through simulations, after quenching. The stripe patterns (a) correspond to the parameters $k=4.2$ and $A=-0.5$. The cells (b) to $k=0.8$ and $A=-0.1$. Finally, the labyrinths (c) to $k=1.2$ and $A=$ -0.8 .

which are now negative and smaller in magnitude. In this case stripe domains, one lattice parameter wide, do not optimize the energy, thus yielding a domain width larger than in the previous case. Finally, when cells are generated [see Fig. 4(b)], both the first- and second-neighbor interactions are ferromagnetic, and all the rest are antiferromagnetic and of much smaller magnitude. Consequently, the system approaches full ferromagnetism and a quenched cell structure results from a frozen metastable spinodal decomposition. Later we will see that this cell structure, as well as the typical eddy structures of the labyrinth case, both originate in the quenching process.

Before going on, it is important to stress the fact that each of the configurations in Fig. 4 originates solely from different Hamiltonian parameters, since both $J_{i j}$ (which is a function of $k$ ) and $A$ do vary. On the other hand, in the experiments each domain pattern is a metastable configuration driven by an external applied bias field $\mathbf{H}$, as mentioned in the Introduction. The effects of $\mathbf{H}$ on the domain patterns and on other physical properties will be addressed in Secs. III C and III D.

\section{B. Characterization}

Our main objective is to contribute toward an understanding of the physical mechanisms responsible for the many qualitatively different configurations that two-dimensional magnetic systems do adopt. Thus, in order to properly characterize and systematize our conclusions, several tools and physical parameters are used to illustrate the results we obtain. Clearly, direct pictures are the most straightforward form of visualization, and consequently we start providing representative snapshots of some of the typical patterns that the system adopts.

To describe the equilibrium state we computed the specific heat $C$, the magnetic susceptibility $\chi$, and the magnetization $M$, as a function of temperature $T$. In addition, in Secs. III C and III D hysteretic and memory effects, respectively, will be presented and discussed.

As already mentioned snapshots provide the most direct perception and they allow one to observe directly the evolution of the system. The patterns displayed in Fig. 5 were obtained during runs at various $T$ values, starting from a high initial temperature, $k_{B} T>\Gamma$, as illustrated in Fig. 5(a), where a cell-like structure develops.

As $T$ is lowered the ordering process is triggered when the critical temperature $T=T_{c}$ is approached [Fig. 5(b) corresponds to $T=0.8$ and a labyrinth pattern does emerge]. Finally, the low temperature [Fig. 5(c) illustrates the $T=0.1$ result] the minimum energy configuration is attained. As expected, the low- $T$ stripe configuration reached in this way exhibits a higher degree of order than the quenched one, illustrated in Fig. 4(c). In other words, the eddy patterns of the quenched labyrinth case are almost washed out by thermal relaxation, evidencing that the latter correspond to a metastable configuration. The experimental counterpart shows a very similar behavior, as reported by Mino et al..$^{15,16}$ for the $\left(\mathrm{Ho}_{1.2} \mathrm{~Tb}_{0.6} \mathrm{Bi}_{1.2}\right) \mathrm{Fe}_{5} \mathrm{O}_{12}$ compound, when the system was relaxed from the quenched configuration under an oscillating magnetic field. This provides support to the fact that our model constitutes a reasonable choice. Moreover, the cell case also corresponds to a metastable pattern, obtained by freezing the spinodal decomposition of a quasiferromagnet. This is confirmed by relaxing the system, which leads to a perfect fully magnetized $(M=1)$ ferromagnet.

The MC thermal relaxation was carried out reducing the temperature from $T=5$ to $T=0$. From the $E$ versus $T$ plot of 
(a)

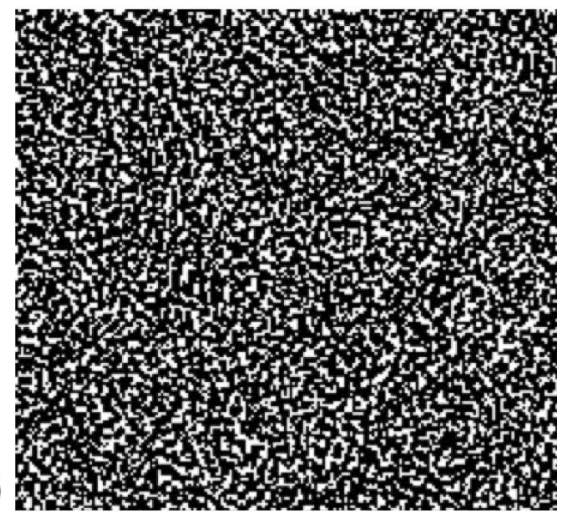

(b)

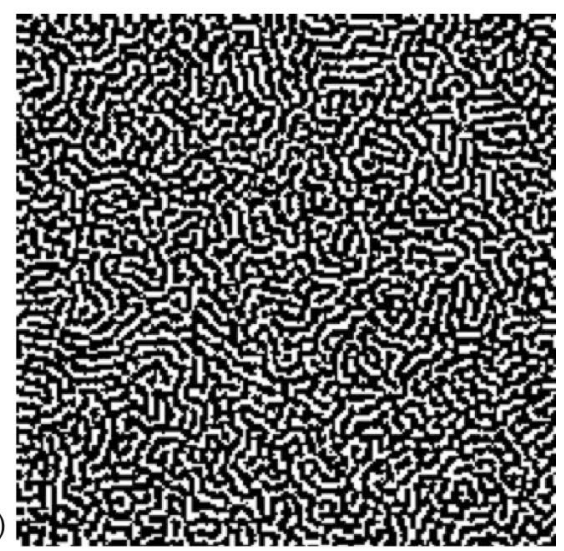

(c)

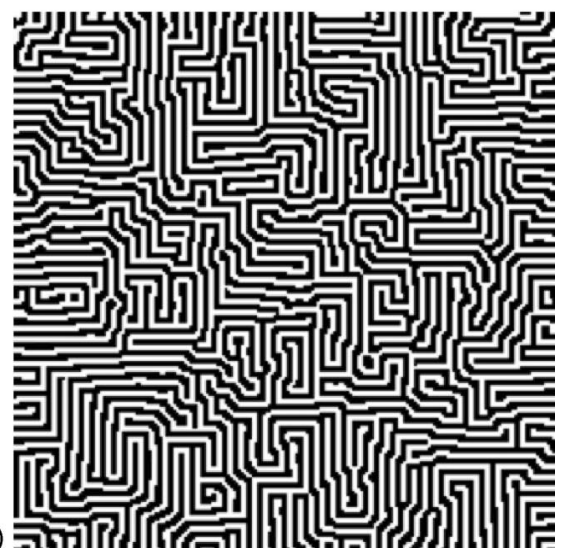

FIG. 5. Snapshots that illustrate the results of simulating the relaxation process for the labyrinth case, starting from a large temperature $T \gg 1$ : at $T=2$ a cell-like structure (a) develops. The ordering process starts around $T=0.8$, as illustrated in (b). Finally, an ordered stripelike pattern is reached at $T=0.1$.

Fig. 6 it is evident that a phase transition takes place at the inflection point. It is apparent as well that the energy sequence is $E_{\text {labyrinth }}>E_{\text {stripe }}>E_{\text {cell }}$, as is verified by direct inspection of Fig. 6. However, the most conclusive evidence for the phase transition is the specific heat $C$, which exhibits a characteristic peak (Fig. 7) that constitutes the signature of the transition. We recall that this curve-and all the others obtained during the relaxation processes-corresponds to an average performed over 20 independent runs. This allows one to appreciate the benefits of such a procedure to attenuate the fluctuations inherent to computations on a relatively

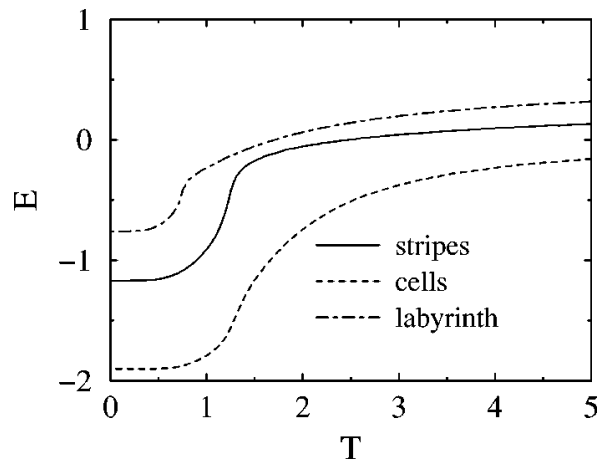

FIG. 6. Energy vs $T$ plots for the three types of patterns. The parameters $k$ and $A$ are the same as in Fig. 4.

small system, of only $200 \times 200$ sites, by comparison with our preliminary results ${ }^{17}$ obtained with only one run. It is observed that the ordering temperatures are of the same order of magnitude as the nearest-neighbor interaction $\Gamma$, which by definition is the same for all the samples. However, the critical temperature $T_{c}$ is larger for the cell configuration, which is mainly ferromagnetic, and lower for the stripe and labyrinth patterns.

For completeness we have also calculated the magnetization fluctuations, i.e., the spin susceptibility $\chi$ at zero field, and the corresponding results are displayed in Fig. 8 for the three main structures we discuss. It is quite apparent that stripes and labyrinths, which order mainly antiferromagnetically, have a relatively low susceptibility, while cells show a value of $\chi$ more than an order of magnitude larger, consistent with their tendency to ferromagnetism. Also, the thermal evolution of the magnetization, $M$ versus $T$, portrayed in Fig. 9 , is a evidence that the stripe and labyrinth structures are antiferromagnetic, as implied by the low-amplitude fluctuations close to $M=0$, with $\langle M\rangle \approx 0$, in sharp contrast with the cell structure results which exhibit a significant ferromagnetic magnetization. All the above provides a picture that is self-consistent and agrees qualitatively with experiment.

\section{Hysteresis cycle}

In order to characterize the system as completely as possible, we also computed the magnetic hysteresis cycles by performing $\mathrm{MC}$ simulations at temperatures $T<T_{c} \sim 1$, adopting the quenched state at zero applied field $(\mathbf{H}=0)$ as

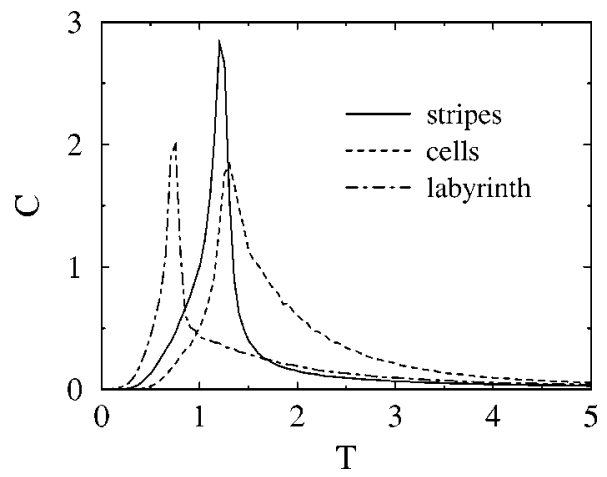

FIG. 7. Specific heat vs $T$ plots for the patterns of Fig. 6 . 


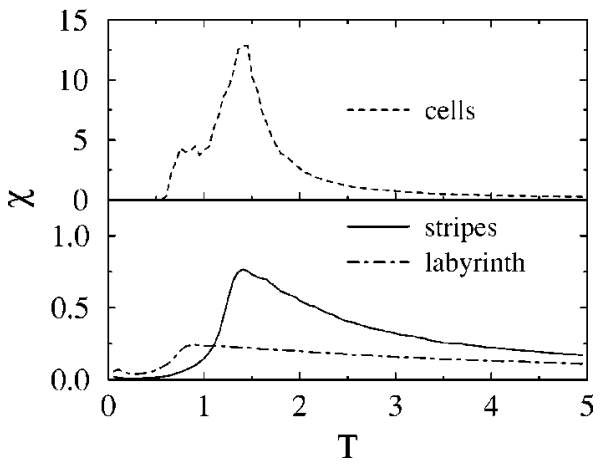

FIG. 8. Susceptibility $\chi$ vs $T$ for the patterns of Fig. 6 .

the initial configuration and thereafter increasing the magnetic field in increments of $\Delta \mathbf{H}=0.01$ (in units of $\Gamma$ ). The magnitude of $M$ was computed averaging over $1000 \mathrm{MCS}$ for each value of the applied field intensity. This process was performed until saturation of the magnetization was achieved, after which the direction of the $\mathbf{H}$ field was reversed and the full hysteresis cycle computed. The results obtained are consistent with the previous discussion. The cell structure exhibits a magnetic hysteresis typical of a soft ferromagnetic system: that is, the system is easily saturated with a relatively low applied field $(\mathbf{H} \approx 0.05)$ and the remanent field is almost as large as the saturation field.

In sharp contrast the stripe structure, illustrated in Fig. 10(a), behaves quite differently. The hysteresis loop consists of two nearly rectangular portions, corresponding to positive and negative magnetizations $M$, shifted away relative to one another. The width of these rectangular portions shrinks considerably as the temperature is increased, but the relative displacement remains nearly constant. Thus, in spite of the fact that the equilibrium configuration is antiferromagnetic, at $T=0.2$ the system remains saturated up to $\mathbf{H} \approx 1.3$, implying that the energy landscape is bumpy and with high-energy barriers. In order to verify the preceding picture we also performed simulations at a higher temperature $T=0.5$. The corresponding hysteresis cycle is also shown in Fig. 10(a), and the dominant antiferromagnetic character is again evident. The total magnetization has a sharp transition from zero to fully saturated, without going through intermediate values; as expected, the width of each of the rectangular portions is significantly smaller than for $T=0.2$ and saturation is obtained for applied fields $\mathbf{H} \approx 0.8$, i.e., not too different from the low-temperature value.

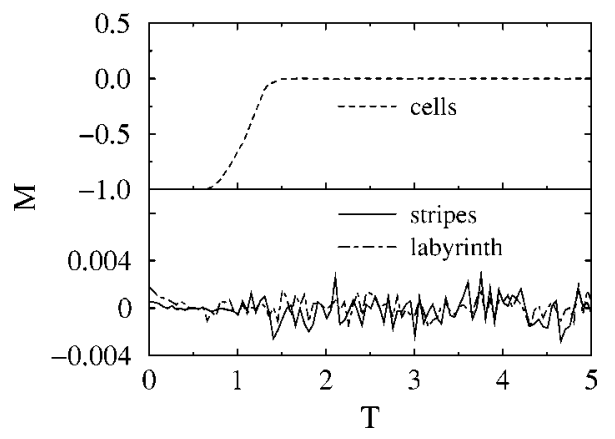

FIG. 9. Magnetization M vs $T$ for the patterns of Fig. 6.
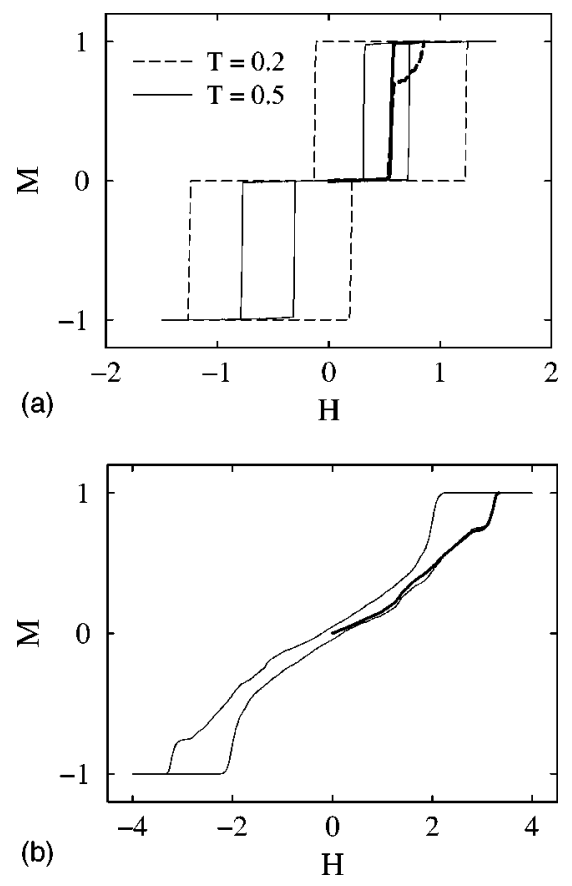

FIG. 10. Hysteresis loops (a) stripes for $T=0.2$ and 0.5 ; (b) labyrinths for $T=0.05$. The virgin curves are marked with thick lines.

On the contrary the labyrinth configuration, plotted in Fig. 10(b), where we represent the low-temperature $T=0.05$ cycle, is rather peculiar. It requires large $\mathbf{H}$ values to reach saturation and the $M$ versus $\mathbf{H}$ cycle is almost reversible (i.e., with very low remanence). Moreover, two different regimes are clearly defined: for low fields the magnetization is essentially a linear function of $\mathbf{H}$, and it is in this region where one can expect to obtain a memory effect. On the contrary, for large applied fields the system saturates, but when $\mathbf{H}$ is decreased it returns to the linear regime for the relatively large $\mathbf{H} \approx 2$ field value.

\section{Memory effect}

Among the results obtained by Albuquerque ${ }^{10}$ perhaps the most striking one is the visualization of the memory effect illustrated in Fig. 11. It consists in the reversible change of the foam topology under a cyclic variation of $\mathbf{H}$. The "foam" observed under an applied field $\mathbf{H}$ in the top image of Fig. 11 evolves into a labyrinth pattern at zero field (middle figure), but the system recovers the initial macroscopic configuration when $\mathbf{H}$ is restored to its original value, as seen in the bottom image of Fig. 11. It has been conjectured that it is the stability of the threefold vertices, which are so abundant in the upper and lower images displayed in Fig. 11, which is responsible for this interesting effect. ${ }^{2,4}$

Unfortunately the memory effect is very difficult to simulate and our efforts in this direction have met with only modest success. In our quest we have investigated the labyrinth ( $k=1.2$ and $A=-0.8)$ and cell $(k=0.8$ and $A=-0.1)$ structures. In both cases we generated the initial structures by first quenching the system and afterwards applying a transverse magnetic field $\mathbf{H}_{t}=0.5$ to favor the growth of a bound- 


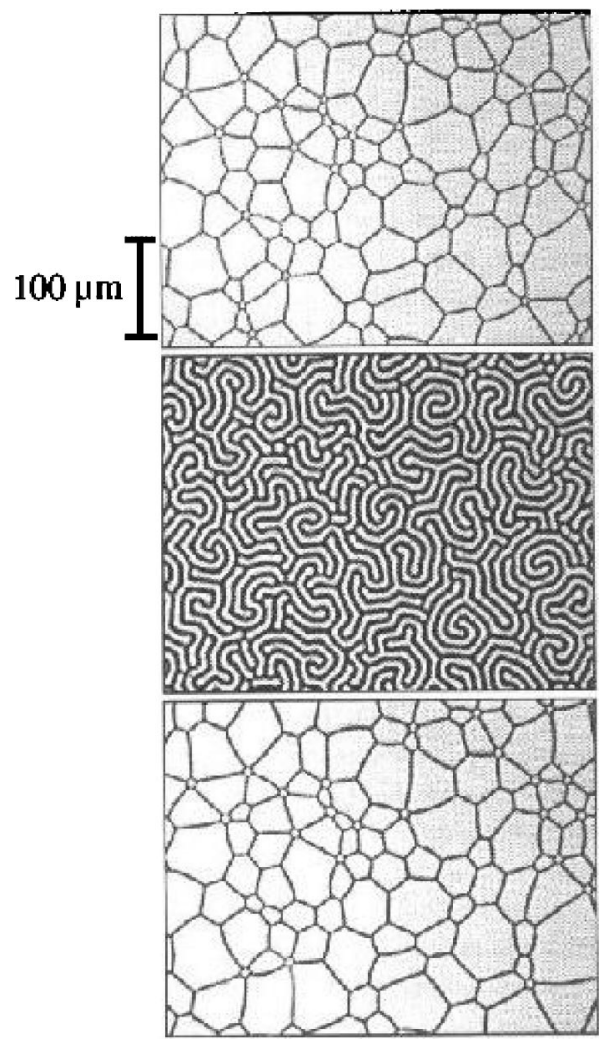

FIG. 11. Memory effect observed by Albuquerque (Ref. 10) under cycling of the applied magnetic field. The top figure corresponds to $\mathbf{H}=78.9$ Oe, the center one to $\mathbf{H}=0$, and the bottom figure again to $\mathbf{H}=78.9$ Oe. The close resemblance of the top and bottom figures is quite remarkable.

ary (actually a "skin") of $S=0$ spins that separates the $S=1$ from the $S=-1$ stripes. It is important to stress that these Bloch walls (or "skins") assemble as a very narrow domain wall (one or two lattice parameters wide) but, in spite of their limited width, they are an important element to generate the memory effect. As a matter of fact, the effect fades away in the absence of these $S=0$ Bloch walls. However, it should also be remarked that, due to the length scale required to display our results in Fig. 12, the "skins" are too narrow to be noticeable.

Following the quenching and the application of $\mathbf{H}_{t}$ a longitudinal field $\mathbf{H}_{l}$, lower than the saturation value, is also applied after which $\mathbf{H}$ is reduced to zero while performing 1000 or more MCS at every field value. The labyrinth structure thus obtained exhibits a sort of memory effect, as can be seen in Figs. 12(a), 12(b), and 12(c), obtained for $\mathbf{H}_{l}=0$, $\mathbf{H}_{l}<1.7$ and $\mathbf{H}_{l}=0$, respectively $\left(\mathbf{H}_{t}=0.5\right.$ is kept constant during the cycle). After several cycles the structure does recover its overall original appearance, thus yielding a genuine memory effect. However, there are differences with the experimental results: both the configuration we start from and the one we arrive at after cycling differ, both as far as the shape of the labyrinths and the width of the walls that separate them are concerned, with experimental observation. On the other hand, in the cell structure case no memory effect is observed, since the system is magnetically very soft. Thus, repeated cycling just yields a fully polarized lattice.

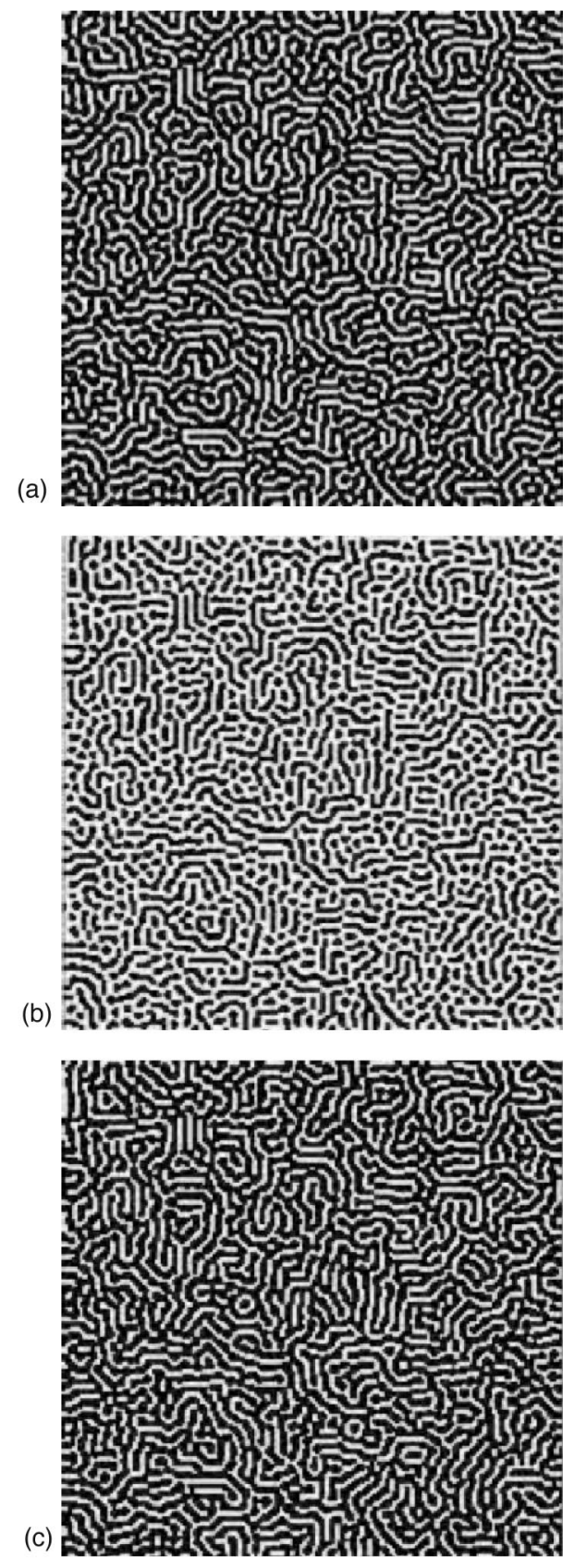

FIG. 12. Simulation results of the memory effect of Fig. 11 for the labyrinth case $\left(\mathbf{H}_{t}=0.5\right)$ : (a) $\mathbf{H}_{l}=0$, (b) $\mathbf{H}_{l}=1.5$, and (c) $\mathbf{H}_{l}$ $=0$.

\section{SUMMARY AND CONCLUSION}

In this paper we model the magnetic relaxation and the formation of magnetic patterns in ultrathin films with anisotropy perpendicular to the film plane. To study this model the Monte Carlo simulation technique was implemented in combination with a generalized two-dimensional classical Isinglike Hamiltonian on a square lattice, including long-range interactions, and transverse $\mathbf{H}_{t}$ and longitudinal $\mathbf{H}_{l}$ applied magnetic fields. For the dipolar interaction we adopted a long-range oscillating coupling that extends all the way to the seventh nearest neighbor.

To present our results we start providing direct snapshots 
of the patterns that develop in the simulations. In addition, and to fully characterize the behavior of the system, we monitor the evolution of the total energy, the magnetization, the average domain size, the specific heat, the magnetic susceptibility, and the hysteresis cycle. Altogether they provide a physical description which is qualitatively in agreement with experimental results, yielding stripe, labyrinth, and cell patterns. The key parameter that determines which type of pattern is generated is the magnitude of $k$ [the argument of the RKKY part of the potential given by Eq. (2.2)] which modulates the oscillations and the relative weight of the long-range interactions. On the other hand, the specific heat versus temperature plot implies the existence of an ordering temperature $T_{c}$ below which pattern formation is observed after quenching the samples.

Finally we address the striking memory effect observed by Albuquerque, ${ }^{10}$ which consists in the reversible change of the foam topology under the cycling of the applied magnetic field. While our model fails to yield the shape and size of the domains observed experimentally, the patterns that evolve when the cycling process is implemented do display the experimentally established memory effect.

\section{ACKNOWLEDGMENTS}

We thank Dr. P. Molho and Dr. M. P. de Albuquerque for enlightening discussions and for allowing us to reproduce some of their results. J.R.I., S.G. and M.K. acknowledge support from the Fundación Andes and O.N. from the Centro Latino-Americano de Física. J.R.I., S.G., and O.N. were supported by CNPq (Brazil) and M.K. by the Fondo Nacional de Investigaciones Científicas y Tecnológicas (FONDECYT, Chile) under Grant No. 8990005.
${ }^{1}$ A. H. Eschenfelder, Magnetic Bubble Technology (SpringerVerlag, New York, 1979).

${ }^{2}$ D. Weaire and N. Rivier, Contemp. Phys. 25, 59 (1984).

${ }^{3}$ K. L. Babcock and R. M. Westervelt, Phys. Rev. Lett. 63, 175 (1989).

${ }^{4}$ K. L. Babcock and R. M. Westervelt, Phys. Rev. A 40, 2022 (1989).

${ }^{5}$ S. K. Kurtz and F. M. A. Carpay, J. Appl. Phys. 51, 5725 (1981).

${ }^{6}$ J. A. Glazier, S. P. Gross, and J. Stavans, Phys. Rev. A 36, 306 (1987).

${ }^{7}$ A. J. Wagner and J. M. Yeomans, Phys. Rev. Lett. 80, 1429 (1998).

${ }^{8}$ L.C. Sampaio, M. P. de Albuquerque, and F. S. de Menezes, Phys. Rev. B 54, 6465 (1996).

${ }^{9}$ M. Portes de Albuquerque and P. Molho, J. Magn. Magn. Mater.
113, 132 (1992).

${ }^{10}$ M. Portes de Albuquerque, Ph.D. thesis, C.N.R.S., Grenoble, 1992.

${ }^{11}$ D. Weaire, F. Bolton, P. Molho, and J. A. Glazier, J. Phys.: Condens. Matter 3, 2101 (1991).

${ }^{12}$ M. E. J. Newman and G.T. Barkema, Monte Carlo Methods in Statistical Physics (Clarendon Press, Oxford, 1999).

${ }^{13}$ J. Hoshen and R. Kopelman, Phys. Rev. B 14, 3428 (1976).

${ }^{14}$ D. Stauffer and A. Aharony, Introduction to Percolation Theory, 2nd ed. (Taylor \& Francis, London, 1998).

${ }^{15}$ M. Mino, S. Miura, K. Dohi, and H. Yamazaki, J. Magn. Magn. Mater. 226-2, 1530 (2001).

${ }^{16} \mathrm{M}$. Mino (private communication).

${ }^{17}$ J. R. Iglesias, O. A. Nagel, S. Gonçalves, and M. Kiwi, J. Magn. Magn. Mater. 226-0, 548 (2001). 